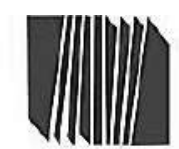

UNIVLRSIDADPEDAGOGICA EXPLR HAENT AL LIB CR TROOR

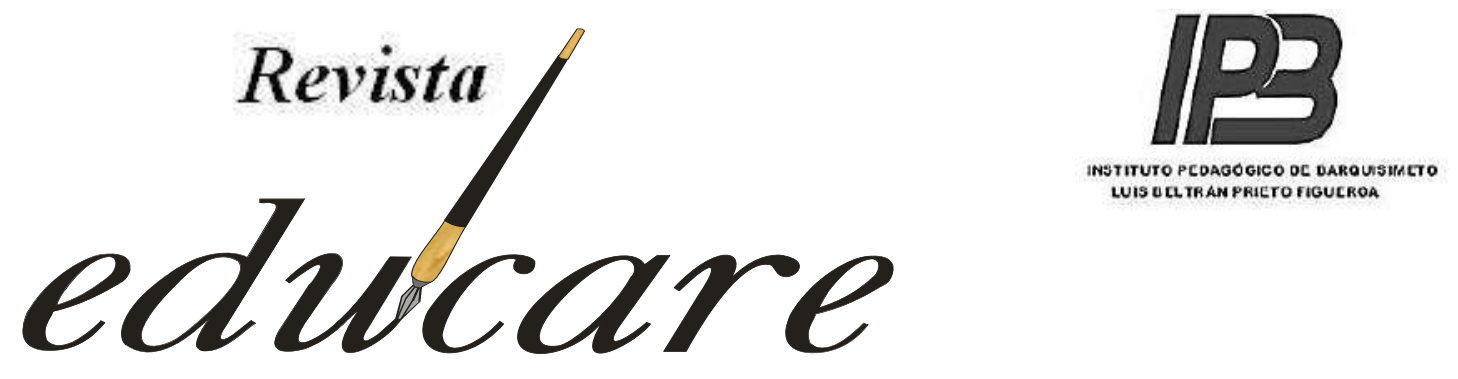

Órgano Divulgativo de la Subdirección de Investigación y Postgrado del Instituto Pedagógico de Barquisimeto "Luis Beltrán Prieto

Figueroa"

BARQUISIUEIO - EDO. LARA - VEAEZUEIA

\title{
ESENCI: ESTRATEGIA DE ENSEÑANZA PARA RESOLUCIÓN DE PROBLEMAS COMPUTACIONALES USANDO ANÁLISIS BASADO EN PROBLEMAS
}

\section{ESENCI: TEACHING STRATEGY FOR COMPUTER PROBLEM SOLUTION USING} PROBLEM BASED ANALYSIS

\section{DEPOSITO LEGAL: ppi201002LA3674 ISSN: 2244-7296}

\author{
María Torres Samuel \\ Maritza Torres Samuel
}

\section{UNIVERSIDAD CENTROCCIDENTAL "LISANDRO ALVARADO" (UCLA)}




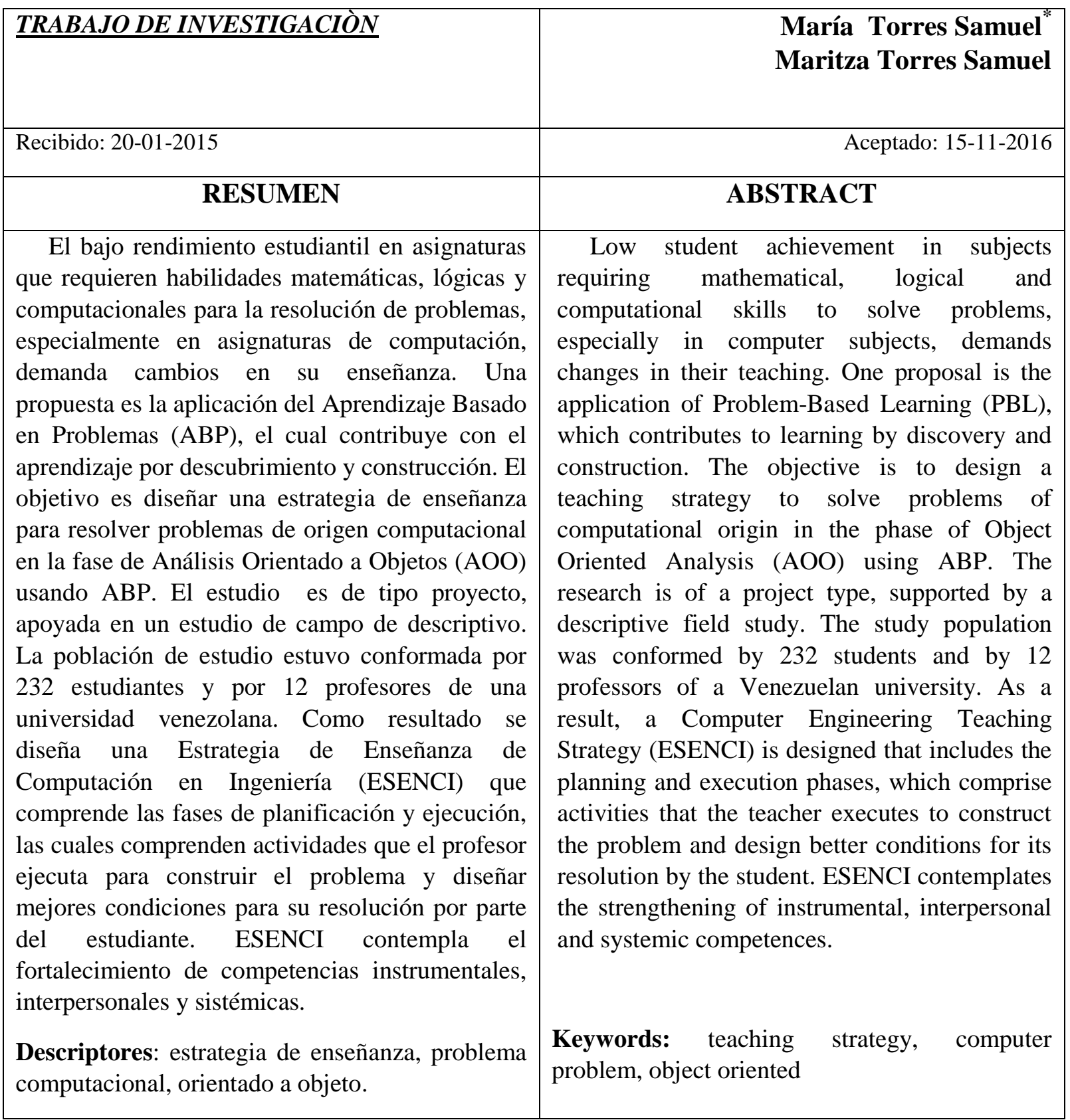

\footnotetext{
"Profesora del Decanato de Ciencias y Tecnología Universidad Centroccidental "Lisandro Alvarado". E-mail: metorres@ucla.edu.ve

"** Profesor Titular Decanato de Ciencias y Tecnología Universidad Centroccidental "Lisandro Alvarado" (UCLA E-mail: mtorres@ucla.edu.ve
} 


\section{INTRODUCCIÓN}

En el contexto internacional, la guía de Computing Curricula CS2013 (2013) de la Asociationfor Computing Machinery (ACM) y el Institute of Electrical and ElectronicsEngineers (IEEE), coinciden en señalar al desarrollo de formas efectivas para resolver problemas computacionalescomo un criterio de desempeño del ingeniero, en el cual demuestre ser capaz de encontrar soluciones robustas y correctas, comprender la idoneidad y complejidad de las soluciones propuestas, así como manejar las restricciones de tiempo y coste. En este sentido, los programas universitarios de ingeniería en informática y afines, requieren incorporar estrategias para la resolución de problemas que le permitan al estudiante ser autónomo en su desempeño.

Al iniciar estudios de computación, los estudiantes se enfrentan a dos dificultades básicas que afectan su rendimiento académico. Según Salgado y otros (2013) una dificultad está asociada a los procesos esenciales que deben llevarse a cabo en la resolución de problemas de programación computacional, los cuales comprenden la modelación matemática y la lógica algorítmica, la otra dificultad se encuentra en las estrategias de enseñanzaaprendizaje (García, 2007).

Resolver un problema a nivel computacional, requiere la comprensión de la situación y conocimientos, especialmente computacionales, matemáticos y sobre el mundo que le rodea (Salgado, Alonso, Gorina y Tardo, 2013). Esteves y otros (2006) afirman que para formarse como programador es necesario adquirir capacidades que van más allá de conocer la sintaxis del lenguaje de programación a utilizar y que tienen que ver con el desarrollo de las habilidades necesarias para la resolución de problemas.

En este sentido Michel (2011) considera necesario revisar el uso de estrategias didácticas enfocadas al aprendizaje de la Programación Orientada a Objetos, y orientar la enseñanza hacia estrategias que promuevan el desarrollo de competencias del perfil profesional y mejore las habilidades lógico-matemáticas en la resolución de problemas computacionales.

Una de las estrategias ampliamente utilizada en la enseñanza es el Aprendizaje Basado en Problemas (ABP) (Morales y Landa, 2004). Este se basa en el principio de usar problemas como punto de partida en la adquisición e integración de los nuevos conocimientos y permite 
al estudiante enfrentar situaciones reales e identificar sus deficiencias en el proceso de aprendizaje. La aplicación de ABP en la enseñanza de la programación, particularmente en el área de resolución de problemas computacionales bajo el enfoque Orientado a Objetos, ofrece una perspectiva novedosa. Al respectoAhumada, Gallegos y Lozano (2012) afirman que las clases dinámicas en la enseñanza de la lógica computacional promueven la interacción con los estudiantes, permitiendo identificar dificultades y planificar el tipo de problema a emplear en la sesión de clases.

Respecto al caso de estudio abordado en el presente trabajo, Introducción a la Computación (IC) del Programa de Ingeniería en Informática del Decanato de Ciencias y Tecnología (DCYT) de la Universidad Centroccidental "Lisandro Alvarado" (UCLA) en Venezuela, su contenido se basa en la resolución de problemas aplicando la metodología Orientado a Objetos $(\mathrm{OO})$ con diversas estructuras computacionales. El objetivo de esta asignatura es dotar al estudiante de los conocimientos fundamentales para iniciarse en el proceso de desarrollo de software, abordando el análisis, diseño e implementación de soluciones a problemas computacionales utilizando estructuras secuenciales, selectivas e iterativas, bajo el enfoque OO. Según datos suministrados por la Dirección de Control de Estudios en dicha universidad el rendimiento académico durante los lapsos 2011-1, 2012-1 y 2012-2 en la asignatura IC se evidenció bajo rendimiento académico, encontrándose un porcentaje de estudiantes aprobados del 55,52\%.

Por lo anterior, atendiendo a una problemática real observada, las propuestas teóricas en materia de enseñanza, y a las dos (2) dificultades principales planteadas por García (2007) y Salgado y otros (2013) respecto a la resolución de problemas de programación computacional y a la estrategia de enseñanza, se plantea en el presente trabajo la incorporación de una estrategia didáctica innovadora y adaptable como $\mathrm{ABP}$, la cual mejore el rendimiento estudiantil permitiéndole al estudiante potenciar sus habilidades matemáticas, lógicas y computacionales en la resolución de problemas computacionales. En este sentido, el presente trabajo tiene como objetivo diseñar una estrategia de enseñanza para resolver problemas de origen computacional, usando ABP, en el Análisis Orientado a Objetos (AOO).

El artículo está estructurado en cuatro (4) secciones. En la primera se realiza la contextualización de la situación de estudio. La segunda abarca el abordaje metodológico de 
la investigación. En la tercera se presentan los resultados obtenidos y la cuarta sección comprende las conclusiones y reflexiones finales.

\section{DESARROLLO}

\section{APRENDIZAJE BASADO EN PROBLEMAS (ABP)}

Una de las estrategias empleadas en la resolución de problemas es el "Aprendizaje Basado en Problemas" (ABP). El ABP es una metodología didáctica surgida en el entorno de las ciencias de la salud a finales de los años sesenta, específicamente en la Universidad de McMaster, Canadá (Arpí, Ávila, Baraldés, Benito, Gutiérrez, Orts, Rigall y Rostan, 2012). "Al trabajar con el ABP, la actividad gira en torno a la discusión de un problema y el aprendizaje surge de la experiencia de trabajar sobre ello, siendo un método que estimula el auto aprendizaje”(Guevara, 2010, p.160).

ABP se caracteriza por un aprendizaje centrado en el estudiante, significativo, lo cual propicia un desarrollo de habilidades y competencias indispensables en su entorno profesional. Se aplica a grupos pequeños de trabajo, que aprenden de manera colaborativa en la búsqueda de resolver un problema planteado por el profesor, para desencadenar así en el aprendizaje auto-dirigido. Sus ventajas han impulsado que sea adoptada en diversas instituciones y especialidades (Morales y Landa, 2004). La Tabla 1 resume los resultados satisfactorios de la aplicación de ABP en el área académica, siendo escasa la aplicación de ABP en la resolución de problemas computacionales bajo el enfoque Orientado a Objetos.

Tabla 1. Relación de investigaciones en el área académica que reportan beneficios por la aplicación de ABP

\begin{tabular}{|c|c|c|c|}
\hline ÁREA/ ASIGNATURA/UNIDAD & AUTORES & AÑO & PAÍS \\
\hline Medicina Tropical & Villalobos & 2006 & Venezuela - Zulia \\
\hline $\begin{array}{c}\text { Gestión y Control de Procesos en Ingeniería } \\
\text { Industrial }\end{array}$ & Sáez y Monsalve & 2008 & Chile \\
\hline Matemáticas - Ingeniería en Informática & Rojas & 2010 & Colombia \\
\hline Computación & Guevara & 2010 & Chile \\
\hline Informática & Martínez y Torres & 2010 & Argentina \\
\hline Estudios Socioculturales & Verde, Cañas, López & 2011 & Cuba \\
\hline
\end{tabular}




\begin{tabular}{|c|c|c|c|}
\hline & y Trujillo & & \\
\hline Derecho & Rué, Font y Cebrián & 2011 & España \\
\hline Sistemas Tributarios & Barco & 2012 & Venezuela - Lara \\
\hline Educación & Jofré y Contreras & 2013 & Chile \\
\hline Programación & $\begin{array}{c}\text { Lovos, González y } \\
\text { Fernández }\end{array}$ & 2014 & Argentina \\
\hline $\begin{array}{c}\text { Fundamentos de Materiales-Arquitectura } \\
\text { Técnica. }\end{array}$ & $\begin{array}{c}\text { Marieta, Andrés y } \\
\text { León }\end{array}$ & 2015 & España \\
\hline
\end{tabular}

Fuente: Elaboración propia

Según Restrepo (2009) los problemas aplicables usando ABP deben poseer el nivel tres de Duch (1996), esto es: requieren capacidad de análisis, síntesis y evaluación, estar relacionados con el mundo real y no toda la información necesaria para resolverlo debe estar contenido en el propio problema. En consecuencia, los estudiantes necesitan hacer investigación, descubrir nuevos materiales y tomar decisiones basados en la información aprendida. Esta clase de problemas puede tener más de una respuesta aceptable y son denotados como no estructurados o abiertos. Es así que para la aplicación de ABP se requiere que el problema posea ciertos criterios básicos que le den mayores probabilidades de éxito (Albanese y Mitchell, 1993).

Romero y García (2008) expresan que los criterios para aplicar ABP en un problema están relacionados con el contenido y su elaboración. Los criterios sobre el contenido de los problemas están relacionados con la actualidad, ser auténticos y relevantes para el estudiante o basado en la vida real o profesional, y ser apropiados al nivel cognitivo y motivacional del mismo. En cuanto a los criterios sobre la elaboración de los problemas, estos comprenden principalmente su complejidad y estructuración. La complejidad está relacionada con el grado de dificultad del problema respecto a la demanda de acciones cognitivas para resolverlos y con la actividad en la memoria de trabajo. Por otra parte la estructuración de un problema determina las formas de llegar a su solución, los parámetros de manipulación, las especificaciones de su situación y la diversidad de la información necesaria para su resolución.

Particularmente para los problemas a ser utilizados en la enseñanza de la computación, se espera que estos presenten diversas soluciones (no estructurados) y no sean fáciles de resolver (complejos).Para la aplicación de ABP se requiere determinar los pasos para organizar la secuencia didáctica. Una propuesta al respecto es el esquema trabajado en la 
Universidad de Lindburg, denominado el método de los siete saltos (SevenJumps) (Restrepo, 2009).

Estos saltos comprenden: 1) Selección del planteamiento del problema; 2) Clarificación de términos a fin que todos los estudiantes comprendan por igual los términos del problema; 3) Análisis del problema para determinar si se trata de un solo problema o si puede dividirse en varios subproblemas para facilitar su solución; 4) Planteamientos tentativos con la formulación de hipótesis explicativas del problema; 5) Determinación de los objetivos de aprendizaje adicionales para dar una mejor solución al problema; 6) Autoestudio individual o tiempo de consultas a expertos o en biblioteca para sustentar las hipótesis formuladas, y finalmente, 7) Discusión final y descarte de hipótesis o explicaciones tentativas.

\section{ESTRATEGIA DE ENSEÑANZA-APRENDIZAJE}

Díaz y Hernández (2002) definen las estrategias de enseñanza-aprendizaje en función del sujeto involucrado. En el primer caso, el énfasis se enfoca en el diseño, programación, elaboración y realización de los contenidos por parte del diseñador o del profesor, y en el segundo caso, la responsabilidad recae sobre el estudiante. Para la conformación de la estrategia propuesta en el presente trabajo, se consideró la investigación de Verde, Cañas, López y Trujillo (2011), quienes formulan una propuesta de estrategia didáctica fundamentada en ABP para la apreciación de las Artes Plásticas, resumida en los siguientes elementos:

1. Objetivo: el propósito o intención de la estrategia sobre los estudiantes.

2. Fundamentación: se detalla la necesidad de implementar la estrategia.

3. Diagnóstico de la situación actual: ubicar las deficiencias observadas en el contexto de la asignatura objeto de estudio.

4. Descripción del estado deseado: los resultados esperados una vez aplicada la estrategia

5. Planeación estratégica: define las acciones a realizar para alcanzar el objetivo propuesto.

La etapa contiene:

5.1 Diagnóstico: está dirigido al diseño y aplicación de los instrumentos para la identificación de las necesidades que poseen los estudiante 
5.2 Planificación: el (los) profesor (es) en conjunto con Jefes de Carreras o de otros profesores que hayan impartido la asignatura, definen medios, recursos y estrategias a utilizar. Inicia con un problema ABP a solucionar por los estudiantes.

5.3 Ejecución: se realiza una introducción al método ABP. Comprende dos (2) fases: En la primera el tutor expone el contenido y organiza los grupos; en la segunda el tutor motiva la ejecución del problema.

5.4 Evaluación: permite valorar cuantitativa y cualitativamente los cambios que ocurren en el aprendizaje de los estudiantes, mediante la evaluación diagnóstica, la autoevaluación y la coevaluación.

6. Instrumentación: contexto donde actuará.

7. Evaluación: se basa en indicadores e instrumentos para medir los resultados de la estrategia propuesta.

En vista que el presente estudio se centra en la estrategia de enseñanza para resolver problemas de origen computacional en la fase de AOO, se seleccionan las acciones Planificación y Ejecución en las cuales el profesor es el actor del proceso para organizar los recursos, definir los actores responsables que participarán, la ubicación, cronograma y diseñar los instrumentos necesarios para recoger y procesar la información generada del proceso de enseñanza.

\section{ANÁLISIS ORIENTADO A OBJETOS (AOO) Y EL PROBLEMA COMPUTACIONAL}

El Análisis Orientado a Objetos es un método utilizado en el desarrollo de software para examinar requisitos desde la perspectiva de las clases y objetos, siendo una forma de modelar el mundo, descubriendo las clases y objetos que forman el vocabulario del dominio del problema. La estructura del problema debe comprender una serie de elementos que le permitan al estudiante identificar las clases y objetos en un nivel dado de abstracción, identificar la semántica de estas clases y objetos, identificar las relaciones entre clases y objetos, e implementar las clases y objetos (Booch, 2001).

El problema computacional dentro del enfoque Orientado a Objetos debe proveer los elementos básicos que lo integran como lo son: las clases, los atributos y métodos. Además, 
utiliza los objetos como elementos fundamentales en la construcción de una solución computacional, para lo cual es necesario clasificar los elementos que se encuentran dentro del problema computacional. La clasificación es un proceso mental que permite agrupar un conjunto de objetos en categorías denominadas clases estableciendo categorías conceptuales o denominaciones abstractas.

\section{METODOLOGÍA}

La investigación está enmarcada en la modalidad de investigación proyectiva (Hurtado, 2010) apoyada en un estudio documental y de campo de carácter descriptivo. La población de estudio está conformada por 232 estudiantes y 12 profesores. Se seleccionó una muestra de 31 estudiantes utilizando muestreo aleatorio proporcional estratificado entre once (11) secciones de la asignatura IC correspondientes al lapso 2013-1. Respecto a la muestra de profesores, se utilizó el método determinístico intencional, seleccionando siete (7) profesores, cantidad que representa más del 50\% de la población. De los siete (7) profesores, cuatro (4) impartieron la asignatura en el lapso 2013-1 y tres (3) impartieron la asignatura en los lapsos (2012-1, 20122). Los profesores fueron seleccionados de acuerdo a su disponibilidad para el momento en que se aplicó el cuestionario.

La investigación comprende las siguientes actividades:

a) Realizar un diagnóstico mediante observación estructurada de la primera prueba parcial de los estudiantes de la asignatura IC durante el Lapso 2013-1, a fin de conocer su desempeño respecto al proceso de resolución de problemas computacionales en la fase de Análisis Orientado a Objetos.

b) Identificar los criterios de ABP esenciales para la construcción y resolución de problemas computacionales y determinar la pertinencia de los pasos en la fase de AOO como parte de la resolución de problemas en la enseñanza de la computación. Esto se logra mediante la aplicación de un cuestionario a los profesores que impartieron la asignatura IC en tres (3) lapsos académicos 2012-1, 2012-2 y 2013-1.

c) Diseñar la estrategia, la cual se construye mediante la revisión documental del método ABP y su aplicación en instituciones de educación superior. De los resultados obtenidos 
del análisis del diagnóstico se obtienen los criterios del problema para aplicar ABP, así como la pertinencia de los pasos en la fase de AOO.

Las técnicas de recolección de datos empleadas para el levantamiento de información fueron la observación directa mediante una ficha de observación de los exámenes parciales donde se aplicó la metodología OO. Se utilizó adicionalmente un cuestionario aplicado al profesor, contentivo de 20 ítems, con escala de tipo Likert y validado por juicio de expertos. A continuación se describen los instrumentos antes mencionados para la recolección de información. Ver Tabla 2.

Tabla 2. Descripción de los instrumentos para la recolección de datos

\begin{tabular}{|c|c|c|c|}
\hline Instrumento & Aplicado a & Descripción & Objetivo \\
\hline $\begin{array}{c}\text { Ficha de } \\
\text { observación }\end{array}$ & $\begin{array}{l}\text { Primera prueba } \\
\text { parcial de los } \\
\text { estudiantes de la } \\
\text { asignatura IC }\end{array}$ & $\begin{array}{l}\text { Estructurado en tres ítems } \\
\text { correspondientes a la puntuación } \\
\text { obtenida por el estudiante en los } \\
\text { tres pasos del AOO: Clase, } \\
\text { Métodos y Relaciones. }\end{array}$ & $\begin{array}{l}\text { Recolectar información sobre } \\
\text { el desempeño del estudiante } \\
\text { en la resolución del problema } \\
\text { planteado en la fase de AOO. }\end{array}$ \\
\hline Cuestionario & $\begin{array}{l}\text { Personal docente de } \\
\text { la asignatura } \text { IC en } \\
\text { Ingeniería } \\
\text { Informática. en }\end{array}$ & $\begin{array}{l}\text { Estructurado en dos secciones } \\
\text { a) El problema computacional, } \\
\text { conformado por } 10 \text { ítems. } \\
\text { b) b. Análisis Orientado a } \\
\text { Objetos, conformado por } 10 \\
\text { ítems. }\end{array}$ & $\begin{array}{l}\text { Identificar los criterios } \\
\text { esenciales en el planteamiento } \\
\text { de un problema computacional } \\
\text { para la aplicación del AOO, } \\
\text { además de determinar las } \\
\text { debilidades en el aprendizaje } \\
\text { del enfoque Orientado a } \\
\text { Objetos }\end{array}$ \\
\hline
\end{tabular}

La confiabilidad del cuestionario aplicado a los profesores fue calculada mediante coeficiente Alpha de Cronbach utilizando el programa NCSS versión 9.0.13, arrojando un valor de $\alpha=0.788807$, el cual según Hernández y otros (2010) presenta confiabilidad fuerte debido a que se ubica en el rango de 0.76 a 0.89 . Para el tratamiento de los datos recolectados en la ficha de observación y el cuestionario se utilizó estadística descriptiva.

Respecto al diseño de la variable Estrategia de Enseñanza de Computación en Ingeniería (ESENCI) se utilizaron los resultados obtenidos de la aplicación de la ficha de observación a las pruebas de los estudiantes, el cuestionario aplicado a los profesores, el método ABP, las estructuras sobre estrategias de enseñanza planteadas por Romero y García (2008), Feo (2010), y Verde y otros (2011), y adicionalmente, el método de los Siete Saltos 
de la Universidad de Lindburg, Holanda (Restrepo, 2009). En la Tabla 3 se presenta el sistema de variables correspondiente a la propuesta de Estrategia de Enseñanza de Computación en Ingeniería (ESENCI).

Tabla 3. Sistema de variable de ESENCI. Estrategia de enseñanza para resolución de problemas computacionales usando análisis basado en problemas bajo el enfoque AOO.

\begin{tabular}{|c|c|c|}
\hline DIMENSIONES & SUB DIMENSIONES & INDICADORES \\
\hline \multirow{4}{*}{$\begin{array}{l}\text { Problema } \\
\text { computacional en } \\
\text { ABP: } \\
\text { Criterios básicos que } \\
\text { debe poseer el } \\
\text { problema para aplicar } \\
\text { ABP }\end{array}$} & \multirow{3}{*}{$\begin{array}{l}\text { Contenido Características } \\
\text { del contenido que deben } \\
\text { motivar al estudiante a } \\
\text { resolver el problema. }\end{array}$} & $\begin{array}{l}\text { Actualidad:referirse a situaciones actuales } \\
\text { contemporáneas. }\end{array}$ \\
\hline & & $\begin{array}{l}\text { Apropiados al nivel cognitivo: ajustarse al nivel de } \\
\text { conocimientos y desarrollo intelectual emocional, } \\
\text { social e intereses de los estudiantes. }\end{array}$ \\
\hline & & $\begin{array}{l}\text { Relevancia:tratar temas del programa del curso } \\
\text { ejercitando lo que el estudiante hará en su vida } \\
\text { profesional. }\end{array}$ \\
\hline & $\begin{array}{l}\text { Elaboración } \\
\text { aprendizaje ropiciar el } \\
\text { descubrimiento } \\
\text { significativo. }\end{array}$ & $\begin{array}{l}\text { Estructuración: presencia o ausencia de los } \\
\text { elementos conocidos del problema. Se espera } \\
\text { problemas que no estén limitados a una única } \\
\text { solución. }\end{array}$ \\
\hline \multirow{10}{*}{$\begin{array}{l}\text { Análisis Orientado a } \\
\text { Objetos: } \\
\text { Forma de modelar el } \\
\text { mundo descubriendo } \\
\text { las clases, objetos que } \\
\text { forman el dominio del } \\
\text { problema }\end{array}$} & \multirow{4}{*}{$\begin{array}{l}\text { Clase: Proceso de } \\
\text { clasificación de los } \\
\text { elementos presentes en el } \\
\text { planteamiento. }\end{array}$} & $\begin{array}{l}\text { Subrayado: Se subrayan en el planteamiento los } \\
\text { verbos, frases con verbos y nombres. }\end{array}$ \\
\hline & & $\begin{array}{l}\text { Identificar clases: Se asignan los atributos y } \\
\text { métodos a las clases identificadas en el } \\
\text { planteamiento. }\end{array}$ \\
\hline & & $\begin{array}{l}\text { Uso de notación para identificadores: Uso de las } \\
\text { notaciones definida para los identificadores. }\end{array}$ \\
\hline & & $\begin{array}{l}\text { Dominio de clase: Comprensión por el estudiante } \\
\text { de la sub dimensión Clase. }\end{array}$ \\
\hline & \multirow{4}{*}{$\begin{array}{l}\text { Métodos: Pasos para la } \\
\text { resolución de las } \\
\text { operaciones aritméticas } \\
\text { del planteamiento del } \\
\text { problema computacional }\end{array}$} & $\begin{array}{l}\text { Definir interfaces de E/S: Definir la secuencia } \\
\text { mediante la cual se dará solución al problema } \\
\text { computacional. }\end{array}$ \\
\hline & & $\begin{array}{l}\text { Operación aritmética: Se desarrolla la operación } \\
\text { aritmética del método. }\end{array}$ \\
\hline & & $\begin{array}{l}\text { Tipo de estructura: Se define el tipo de estructura } \\
\text { del método, esto es, si es secuencial o selectiva. }\end{array}$ \\
\hline & & $\begin{array}{l}\text { Dominio de métodos: Comprensión del estudiante } \\
\text { de la sub dimensión Métodos. }\end{array}$ \\
\hline & \multirow{2}{*}{$\begin{array}{l}\text { Relaciones: Establecer el } \\
\text { tipo de asociación y } \\
\text { cardinalidad entre clases }\end{array}$} & $\begin{array}{l}\text { Relaciones entre clases: Se establece el tipo de } \\
\text { asociación y cardinalidad entre clases. }\end{array}$ \\
\hline & & $\begin{array}{l}\text { Dominio de relaciones: Comprensión por parte del } \\
\text { estudiante de la sub dimensión Relaciones. }\end{array}$ \\
\hline
\end{tabular}




\begin{tabular}{|c|c|c|}
\hline \multirow{3}{*}{$\begin{array}{c}\begin{array}{c}\text { Estrategia de } \\
\text { enseñanza: }\end{array} \\
\text { Encuentro pedagógico } \\
\text { que se realiza } \\
\text { mediante un diálogo } \\
\text { didáctico pertinente a } \\
\text { las necesidades de los } \\
\text { estudiantes }\end{array}$} & $\begin{array}{l}\text { Planificación: Definición } \\
\text { de medios, recursos y } \\
\text { estrategias a utilizar por } \\
\text { parte del (los) profesor } \\
\text { (es) conjuntamente con } \\
\text { Directores de Programas } \\
\text { Se inicia con un problema } \\
\text { ABP para ser solucionado } \\
\text { por los estudiantes }\end{array}$ & $\begin{array}{l}\text { Definición del objetivo: Identificar las } \\
\text { competencias, conocimientos o habilidades que se } \\
\text { esperan desarrollar y para los cuales se diseña el } \\
\text { problema }\end{array}$ \\
\hline & \multirow{2}{*}{$\begin{array}{l}\text { Ejecución: Comprende la } \\
\text { exposición de contenidos } \\
\text { y la ejecución de la } \\
\text { resolución del problema }\end{array}$} & $\begin{array}{l}\text { Exponer el contenido del tema: Se definen } \\
\text { objetivos, se aclaran los términos y se analiza el } \\
\text { problema en función de los pasos para la fase de } \\
\text { AOO. }\end{array}$ \\
\hline & & $\begin{array}{l}\text { Cierre de la actividad: Se cierra la actividad } \\
\text { considerando todas las soluciones encontradas al } \\
\text { problema. }\end{array}$ \\
\hline
\end{tabular}

\section{RESULTADOS}

\section{Desempeño de los estudiantes en el proceso de resolución de problemas} computacionales en la fase de Análisis Orientado a Objetos

Respecto al dominio demostrado por los estudiantes de Introducción a la Computación en las pruebas escritas respecto a la resolución de problemas computacionales en la fase de Análisis Orientado a Objetos, se encontró lo siguiente:

Dominio de clases: Involucra subrayar los nombres, verbos y frases con verbos, identificar las clases asignándole atributos y métodos, haciendo uso de la notación establecida para identificadores. El 62, 12\% de los estudiantes aprobaron este paso de la fase de AOO.

Considerando los datos recopilados respecto a las calificaciones de los estudiantes cursantes de las once secciones de la asignatura Introducción a la Computación en el lapso 2013-1, se obtuvo que el mayor porcentaje de aprobados en la fase de análisis se observó en el paso Relaciones entre las Clases, seguido de Identificación de Clases, Atributos y Métodos; por último, con el menor dominio demostrado, el Desarrollo de Métodos. En general el $57.85 \%$ de los estudiantes aprueban la fase de análisis, sin embargo respecto al Desarrollo de Métodos, el cual comprende la resolución aritmética del problema aplicando lógica computacional y la definición del tipo de estructura del método (secuencial, selectivo), se presenta un bajo nivel de aprobación (46.97\%). En la Figura 1 muestra el dominio observado por los estudiantes durante la fase de AOO. 


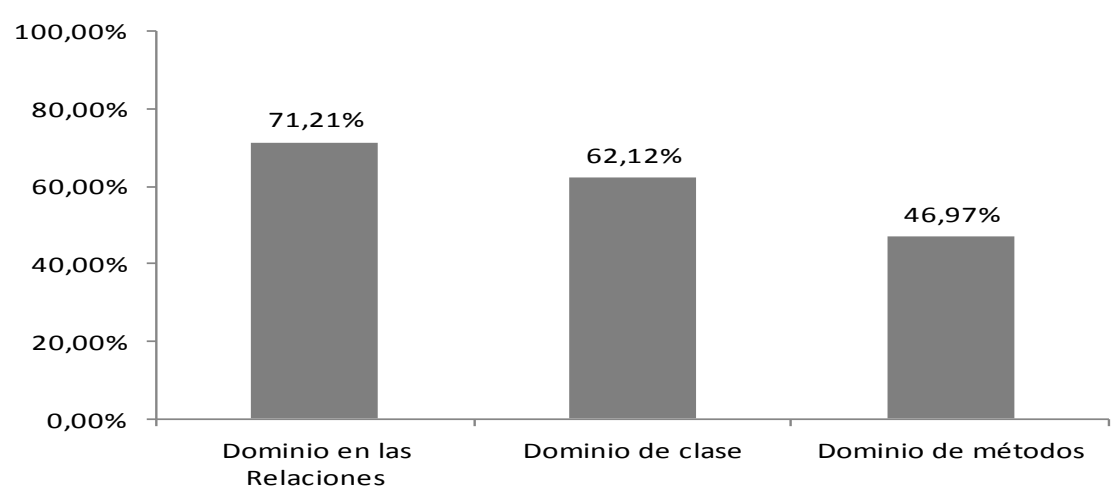

Figura 1: Dominio observado por los estudiantes durante la fase de AOO

Dominio de métodos: Comprende definir interfaces de Entrada y Salida, desarrollar operaciones aritméticas del planteamiento e indicar el tipo de estructura en el desarrollo de la operación matemática. El 46, 97\% de los estudiantes aprobaron este paso en la fase de AOO.

Dominio en las Relaciones: Comprende establecer las relaciones entre las clases, indicando la asociación y cardinalidad. El 72,21\% de los estudiantes aprobaron este paso en la fase de AOO.

\section{Identificación de los criterios de ABP esenciales para la construcción y resolución} de problemas computacionales.

Los criterios de ABP considerados como esenciales por parte de los profesores para la construcción y resolución de los problemas computacionales son Relevancia profesional del problema y Apropiado al nivel cognitivo del estudiante. La Estructuración del problema y la Actualidad del tema son consideradas importantes por el 50\% de los profesores. Ver Figura 2. 

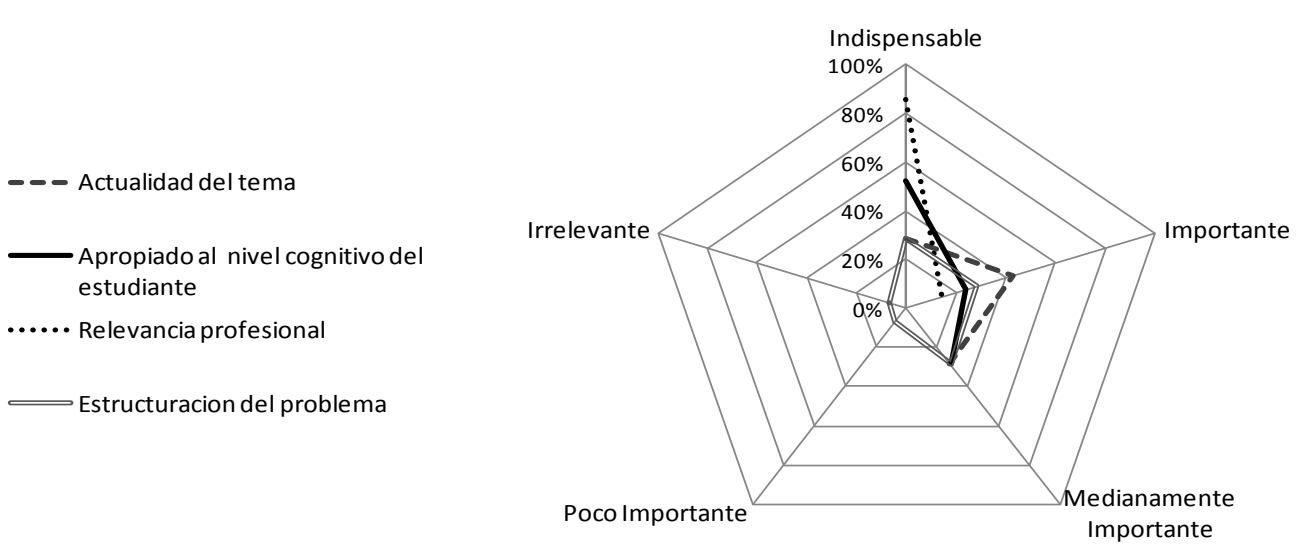

Figura 2. Apreciación de los profesores respecto a los criterios de ABP que son esenciales para la construcción y resolución de problemas computacionales

Determinación de los pasos esenciales en la fase de AOO para la resolución de problemas en la enseñanza de la computación.

Para un alto porcentaje de profesores, en el AOO son esenciales, los pasos relativos a identificar clases, uso de notación para los identificadores, definir interfaces de E/S y elaborar las operaciones aritméticas (ver Figura 3).

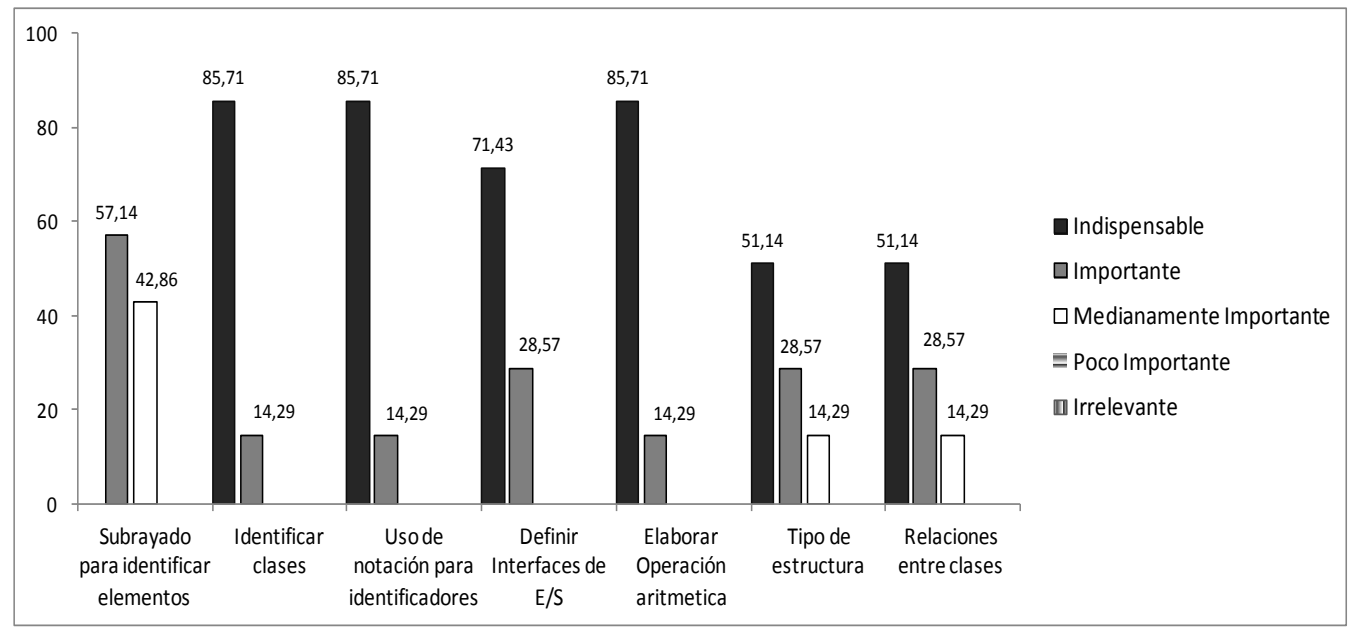

Figura 3: Apreciación de los profesores respecto a los pasos esenciales en la fase de AOO para la construcción y resolución de problemas computacionales 
Por otra parte, los profesores señalaron en el cuestionario que entre las debilidades más notorias observadas en el aprendizaje del enfoque Orientado a Objetos de los estudiantes de Introducción a la Computación se tienen las siguientes: baja comprensión del dominio de los problemas, falta de abstracción para manejar los problemas, bajo dominio matemático, carencias en el proceso de lectura e interpretación de los problemas, el estudiante se encuentra centrado en la herramienta de software a utilizar más que en el dominio de la lógica computacional. Algunas de estas coinciden con causas identificadas por otras investigaciones (Zapata y Flores, 2008; Álvarez y Ruiz, 2010) realizadas en el área de la resolución de problemas.

Finalmente, respecto a la estrategia de enseñanza, se encontró que los profesores coincidieron en apreciar como indispensables los aspectos asociados a la definición del objetivo y exponer el contenido del tema

\section{ESENCI: ESTRATEGIA DE ENSEÑANZA DE COMPUTACIÓN EN INGENIERÍA}

La estrategia propuesta se basa en las estructuras planteadas por Feo (2010), Romero y García (2008), Verde y otros (2011) y el Método de los Siete Saltos de la Universidad de Lindburg, Holanda, y los resultados obtenidos en la presente investigación. ESENCI involucra los pasos esenciales en la enseñanza de resolución de problemas computacionales en ingeniería basada en el método ABP y contribuye a la vinculación teoría-prácticainvestigación presente a lo largo del desempeño profesional del egresado del área computacional, además de fomentar el auto aprendizaje y el trabajo en equipo.

Adicionalmente, permite al profesor planificar la estrategia de enseñanza para la resolución de problemas basado en $\mathrm{ABP}$ bajo el enfoque Orientado a Objetos en la fase de AOO empleando estructuras secuenciales y selectivas. Desde la perspectiva del profesor, ESENCI comprende dos etapas: Planificación y Ejecución.

La Etapa de Planificación de ESENCI (ver Tabla 4) define las acciones a realizar por el profesor y las condiciones necesarias para que los estudiantes aborden y resuelvan el problema basado en ABP. La estructura del problema en ABP se apoya en los criterios sobre 
contenido y elaboración de Romero y García (2008), seleccionando mediante el diagnóstico de la presente investigación los criterios esenciales a ser aplicados en el problema computacional. Una vez integrado los criterios esenciales de ABP al planteamiento del problema computacional se aplica la metodología Orientada a Objetos en la fase de AOO. La Etapa de Ejecución de ESENCI (ver Tabla 5) consiste en la aplicación de la estrategia en el aula de clases, utilizando para ello el Método de los Siete Saltos.

Tabla 4. ESENCI. Etapa I, Planificación.

\section{ESENCI: ETAPA 1. PLANIFICACIÓN}

Define las acciones a realizar por el profesor y las condiciones necesarias para que los estudiantes aborden el problema basado en ABP y lo resuelvan.

\begin{tabular}{|c|c|}
\hline PASOS & DESCRIPCIÓN \\
\hline 1.Definir objetivos & $\begin{array}{l}\text { Comprende la definición de competencias, conocimientos } \\
\text { o habilidades que se quieren desarrollar y para los cuales } \\
\text { se diseña el problema. }\end{array}$ \\
\hline $\begin{array}{l}\text { 2. Criterios sobre el contenido de los } \\
\text { problemas }\end{array}$ & $\begin{array}{l}\text { Los criterios son elementos que están presentes en el } \\
\text { planteamiento del problema. }\end{array}$ \\
\hline 2.1 Criteric & $\begin{array}{l}\text { Los criterios indispensables presentes como características } \\
\text { en el problema computacional. }\end{array}$ \\
\hline $\begin{array}{l}\text { 2.1.1 Apropiado al nivel cognitivo del } \\
\text { estudiante }\end{array}$ & $\begin{array}{l}\text { El contenido del problema debe ajustarse al nivel de } \\
\text { conocimientos y desarrollo intelectual del estudiante. }\end{array}$ \\
\hline $\begin{array}{l}2.1 .2 \text { El ámbito del problema es } \\
\text { conocido por el estudiante }\end{array}$ & $\begin{array}{l}\text { Se relaciona con la familiaridad del problema. La temática } \\
\text { debe ser conocida por el estudiante o tener cierta } \\
\text { experiencia previa sobre el tema. }\end{array}$ \\
\hline $\begin{array}{l}2.1 .3 \text { El problema está relacionado con } \\
\text { el futuro ámbito profesional }\end{array}$ & $\begin{array}{l}\text { Los problemas deben tratar temas del programa del curso } \\
\text { ejercitando lo que el estudiante tendrá que hacer en su } \\
\text { vida profesional. }\end{array}$ \\
\hline 2.2 Criterios no esenciales & Los criterios no indispensable, opcionales. \\
\hline 2.2.1 Relacionado c & $\begin{array}{l}\text { El problema está asociado a situaciones actuales o } \\
\text { contemporáneas }\end{array}$ \\
\hline $\begin{array}{l}\text { 2.2.2 Relacionado con sucesos reales del } \\
\text { acontecer local, nacional o internacional }\end{array}$ & $\begin{array}{l}\text { El problema está relacionado a acontecimientos que } \\
\text { sucedieron a nivel local, nacional o internacional. }\end{array}$ \\
\hline $\begin{array}{l}\text { 3. Criterios sobre la elaboración del } \\
\text { problema }\end{array}$ & $\begin{array}{l}\text { Comprende la estructura y complejidad del problema el } \\
\text { cual define el grado de dificultad. }\end{array}$ \\
\hline 3.1 Estructura & La estructura define el grado de dificultad del problema \\
\hline 3.1.1 Criterio esencial & Criterios indispensables sobre la estructura del problema \\
\hline $\begin{array}{l}\text { 3.1.1.1 El problema debe tener varias } \\
\text { soluciones posibles }\end{array}$ & $\begin{array}{l}\text { El problema posee soluciones múltiples o divergentes. } \\
\text { Existen diversas vías para llegar a la solución }\end{array}$ \\
\hline 3.2.1 Criterio no esencial & Criterios no esenciales sobre la estructura del problema \\
\hline $\begin{array}{l}\text { 3.2.1.1 El problema debe tener poca } \\
\text { información o datos para resolverlo. }\end{array}$ & $\begin{array}{l}\text { La información necesaria para resolver el problema no } \\
\text { está contenida en el texto del problema o es escasa. }\end{array}$ \\
\hline 4.Determinar la disponibilidad de & Organización del lugar: aula, laboratorio y medios para \\
\hline
\end{tabular}




\begin{tabular}{|l|l|}
\hline recursos para resolver el problema & $\begin{array}{l}\text { levar a cabo la actividad: Recursos bibliográficos, } \\
\text { internet, etc. }\end{array}$ \\
\hline $\begin{array}{l}\text { 5.Definir el tiempo y momento } \\
\text { oportuno para la resolución del } \\
\text { problema por parte de los } \\
\text { estudiantes }\end{array}$ & $\begin{array}{l}\text { Se determina el tiempo que deben invertir los estudiantes } \\
\text { en la solución del problema y el momento en que se } \\
\text { ejecutará la estrategia. }\end{array}$ \\
\hline
\end{tabular}

Tabla 5. ESENCI. Etapa II. Ejecución.

\begin{tabular}{|c|c|}
\hline \multicolumn{2}{|c|}{$\begin{array}{l}\text { ESENCI: ETAPA II. EJECUCIÓN } \\
\text { Define los pasos necesarios para resolver el problema siguiendo el método de los siete saltos en ABF }\end{array}$} \\
\hline PASOS (Saltos) & DESCRIPCIÓN \\
\hline 1. Plantear el problema & $\begin{array}{l}\text { Se selecciona el problema del banco de problemas } \\
\text { preparados por el comité curricular y se definen las } \\
\text { competencias a alcanzar. }\end{array}$ \\
\hline $\begin{array}{l}\text { 2. Clarificar los términos: } \\
\text { Aclarar conceptos y términos relativos al } \\
\text { problema seleccionado }\end{array}$ & $\begin{array}{l}\text { - Se define los términos empleados en el planteamiento } \\
\text { del problema. } \\
\text { - Se establece que todos los estudiantes comprendan } \\
\text { por igual los términos del problema. }\end{array}$ \\
\hline $\begin{array}{l}\text { 3. Analizar el problema en función } \\
\text { de su solución, cumpliendo los } \\
\text { pasos de la fase de AOO }\end{array}$ & $\begin{array}{l}\text { Se determina si se trata de un solo problema o si } \\
\text { puede dividirse en varios sub problemas. }\end{array}$ \\
\hline 3.1 Pasos esenciales & Los pasos esenciales para la resolución del problema. \\
\hline 3.1.1 Identificar clases & $\begin{array}{l}\text { Se definen las clases en el enfoque OO que están } \\
\text { presentes en el problema. }\end{array}$ \\
\hline 3.1.2 Asignar atributos a las clases & Se asignan las características asociadas a la clase. \\
\hline 3.1.3 Asignar métodos a las clases & $\begin{array}{l}\text { Se asigna(n) el (los) comportamiento(s) asociado(s) a } \\
\text { la clase. }\end{array}$ \\
\hline $\begin{array}{l}\text { 3.1.4 Desarrollar operaciones aritméticas } \\
\text { del planteamiento }\end{array}$ & $\begin{array}{l}\text { Se desarrolla la operación aritmética de los métodos } \\
\text { identificados que requieren operaciones matemáticas. }\end{array}$ \\
\hline $\begin{array}{l}\text { 3.1.5 Definir interfaces de entrada } y \\
\text { salida }\end{array}$ & $\begin{array}{l}\text { Se define la secuencia mediante la cual se dará } \\
\text { solución al problema computacional en función de } \\
\text { entradas y salidas. }\end{array}$ \\
\hline $\begin{array}{l}\text { 3.1.6 Indicar el tipo de estructura } \\
\text { (secuencial, selectiva) en el desarrollo } \\
\text { del método }\end{array}$ & $\begin{array}{l}\text { Se identifica el tipo de estructura a utilizar en el } \\
\text { método: secuencia o selectivo. }\end{array}$ \\
\hline 3.1.7 Definir relaciones entre clases & $\begin{array}{l}\text { Se establece el tipo de asociación y cardinalidad entre } \\
\text { clases. }\end{array}$ \\
\hline 3.2. Pasos no esenciales & $\begin{array}{l}\text { Pasos no indispensables para la resolución del } \\
\text { problema. }\end{array}$ \\
\hline $\begin{array}{l}\text { 3.2.1 Utilizar la técnica del subrayado } \\
\text { para identificar elementos en el } \\
\text { problema computacional }\end{array}$ & $\begin{array}{l}\text { Se subraya en el planteamiento los verbos, frases con } \\
\text { verbos y nombres, los cuales dan origen a clases, } \\
\text { atributos y métodos. }\end{array}$ \\
\hline $\begin{array}{l}\text { 3.2.2. Hacer uso de las notaciones } \\
\text { establecidas para identificadores }\end{array}$ & $\begin{array}{l}\text { Uso de las normas definidas para denotar para los } \\
\text { identificadores. }\end{array}$ \\
\hline $\begin{array}{l}\text { 4. Incentivar la formulación de } \\
\text { hipótesis }\end{array}$ & $\begin{array}{l}\text { Se incentiva a los participantes a formular hipótesis a } \\
\text { partir de la preparación teórica que disponen. }\end{array}$ \\
\hline 5. Determinar los temas a consultar & $\begin{array}{l}\text { Se determina que temáticas es preciso consultar o } \\
\text { profundizar para dar mejor solución al problema. }\end{array}$ \\
\hline 6. Determinar el tiempo necesario & Se determina el tiempo de consulta a expertos, \\
\hline
\end{tabular}




\begin{tabular}{|l|l|}
\hline $\begin{array}{l}\text { para el estudio de temas a } \\
\text { profundizar }\end{array}$ & $\begin{array}{l}\text { biblioteca o bibliografía para sustentar las hipótesis } \\
\text { formuladas. }\end{array}$ \\
\hline $\begin{array}{l}\text { 7. Realizar cierre de la actividad } \\
\text { considerando todas las soluciones } \\
\text { encontradas al problema }\end{array}$ & $\begin{array}{l}\text { Se cierra la actividad y se descartan las hipótesis o } \\
\text { explicaciones tentativas producto del cuarto salto. }\end{array}$ \\
\hline
\end{tabular}

La estrategia propuesta, en comparación con las revisadas en este trabajo, coinciden en otorgar una vital importancia a la adecuada formulación de los problemas para el aprendizaje de los estudiantes respecto a su resolución, prestando especial atención a aspectos esenciales como son su estructuración y complejidad, privilegiando la construcción del conocimiento por parte del estudiante (Romero y García, 2008; Restrepo, 2009). Por otra parte, la incorporación de pasos propios del Análisis Orientado a Objeto, hacen de ella una estrategia novedosa y de aplicación específica para la enseñanza de la programación en programas de computación.

\section{Bondades de la estrategia ESENCI}

La estrategia propuesta orienta al profesor en la planificación de los aspectos esenciales a evaluar en el problema computacional, de acuerdo a los criterios relacionados con su contenido y elaboración, siguiendo una secuencia detallada del proceso de construcción que guía a la resolución por parte del estudiante.

La aplicación de la estrategia propuesta fomenta la independencia del estudiante hacia el profesor en relación al compromiso que el primero adquiere en la realización de su propio aprendizaje. Esta experiencia se puede extender a otras unidades temáticas del Programa Instruccional de IC por ser una asignatura de naturaleza teórico-práctica.

Por otra parte, ESENCI contribuye con el desarrollo de competencias, ajustado a lo establecido por el Sistema de Evaluación y Acreditación (SEA), el cual establece como competencia el desempeño social basado en conocimientos, habilidades, aptitudes de una persona dentro de una actividad específica (OPSU, 2004). Adicionalmente, mediante la aplicación de la estrategia propuesta se fortalecen las competencias genéricas instrumentales, interpersonales y sistémicas establecidas en el Manual de Directrices para la Formulación del Perfil Profesional bajo Enfoque de Competencias (UCLA, 2005), ver Tabla 6. 
Tabla 6. Competencia genérica que abarca ESENCI

\begin{tabular}{|c|c|c|}
\hline COMPETENCIA & ATRIBUTOS & ESENCI \\
\hline \multirow{9}{*}{$\begin{array}{l}\text { Competencias } \\
\text { Instrumentales }\end{array}$} & - Capacidad de análisis y síntesis. & $\sqrt{ }$ \\
\hline & - Capacidad de organizar y planificar. & $\checkmark$ \\
\hline & - Resolución de problemas. & $\sqrt{ }$ \\
\hline & $\begin{array}{l}\text { - Habilidades de gestión de la profesión (buscar y analizar } \\
\text { información). }\end{array}$ & $\sqrt{ }$ \\
\hline & - Habilidades básicas del manejo de un ordenador & \\
\hline & - Conocimientos generales de la profesión. & $\checkmark$ \\
\hline & - Conocimiento de una segunda lengua & \\
\hline & - Comunicación oral y escrita en la propia lengua & \\
\hline & - Toma de decisiones. & $\checkmark$ \\
\hline \multirow{8}{*}{$\begin{array}{c}\text { Competencias } \\
\text { Interpersonales }\end{array}$} & - Capacidad crítica y autocrítica. & $\checkmark$ \\
\hline & - Trabajo en equipo. & $\sqrt{ }$ \\
\hline & - Habilidades interpersonales. & $\checkmark$ \\
\hline & - Capacidad para trabajar en un equipo Interdisciplinar. & $\checkmark$ \\
\hline & - Compromiso ético & \\
\hline & - Apreciación de la diversidad y multiculturalidad & \\
\hline & - Habilidad de trabajar en un contexto internacional & \\
\hline & - Capacidad para comunicarse con expertos de otras áreas. & $\sqrt{ }$ \\
\hline \multirow{12}{*}{$\begin{array}{l}\text { Competencias } \\
\text { Sistémicas }\end{array}$} & -Capacidad de aplicar los conocimientos en la práctica. & $\sqrt{ }$ \\
\hline & -Habilidades de investigación & $\sqrt{ }$ \\
\hline & -Capacidad de aprender & $\checkmark$ \\
\hline & -Capacidad de adaptarse a nuevas situaciones & $\sqrt{ }$ \\
\hline & -Diseño y gestión de proyectos & \\
\hline & -Iniciativa y espíritu emprendedor & $\checkmark$ \\
\hline & -Capacidad para generar nuevas ideas (creatividad) & $\checkmark$ \\
\hline & -Liderazgo & \\
\hline & -Conocimientos de culturas y costumbres de otros países & \\
\hline & -Habilidad para trabajar de forma autónoma & $\sqrt{ }$ \\
\hline & -Preocupación por la calidad & \\
\hline & -Motivación al logro & $\sqrt{ }$ \\
\hline
\end{tabular}

Fuente: UCLA (2005), adaptado por las autoras.

La Tabla 6 muestra que ESENCI abarca el 66,67\% de las competencias instrumentales, $50 \%$ de las competencias interpersonales y 66,67\% de las competencias sistémicas. En general, ESENCI cumple el 58\% de dichas competencias.

\section{Ejemplo de aplicación de la propuesta ESENCI}

A continuación se ilustra la aplicación de la estrategia ESENCI mediante un ejemplo donde se describe cada uno de los pasos que comprenden las dos etapas de la estrategia: Planificación y Ejecución. 


\section{Etapa I: Planificación}

\section{Planteamiento del problema}

Una asignatura posee cuatro evaluaciones: Tres evaluaciones parciales de 25 puntos cada una y una evaluación continua de 25 puntos. Conociendo la cédula de identificación del estudiante y las cuatro calificaciones obtenidas, calcule la calificación final en escala de 100 y 20 puntos, muestre además si el estudiante reprobó, sustituye o aprobó la asignatura. En caso que el estudiante requiera sustituir la prueba debido a una baja calificación obtenida, indique cuál de las evaluaciones parciales debe presentar. Considere que las calificaciones obtenidas en las evaluaciones parciales son diferentes entre sí.

\section{Pasos:}

1. Definir objetivos: Comprende la definición de competencias, conocimientos o habilidades que se quieren desarrollar para los cuales se diseña el problema.

\section{Competencias:}

Competencias Instrumentales:

- Capacidad de análisis y síntesis

- Capacidad de organizar y planificar

- Habilidades de gestión de la profesión (buscar y analizar información)

- Conocimientos generales de la profesión

- Toma de decisiones

Competencias Interpersonales:

- Trabajo en equipo

- Habilidades interpersonales

\section{Etapa II: Ejecución}

\section{Plantear el problema}

Se plantea el problema seleccionado en la Planificación y se detallan las competencias y conocimientos o habilidades a lograr.

\section{Clarificar los términos}

Se aclaran los conceptos y términos relativos al problema seleccionado, como por ejemplo la calificación requerida para que un estudiante pueda optar a sustituir la prueba. 


\section{Analizar el problema en función de su solución, cumpliendo los pasos de la fase de} AOO

Pasos esenciales:

- Clase

Identificar clases, asignar atributos y métodos a las clases.

Usar notación para identificadores.

- Métodos

Definir interfaces de Entrada/Salida.

Desarrollar operaciones aritméticas de los métodos que lo requieran.

\section{- Relaciones}

Definir la relación entre clases.

\section{Incentivar la formulación de hipótesis}

Se discuten las posibles soluciones al problema, motivando su solución.

\section{Determinar los temas a consultar}

Revisión de los elementos básicos que conforman un algoritmo, revisión del material de clases, revisión del Reglamento de evaluación y rendimiento estudiantil de la UCLA.

\section{Determinar el tiempo necesario para el estudio de temas a profundizar}

La entrega de la fase de AOO se realizará en la siguiente sesión de clases.

\section{Realizar cierre de la actividad considerando todas las soluciones encontradas al} problema.

El profesor realiza el cierre de la actividad, considerando todas las formas de resolver el problema encontradas por cada uno de los equipos de trabajo.

\section{CONCLUSIONES}

La Estrategia de Enseñanza de Computación en Ingeniería (ESENCI) se diseña como respuesta a las deficiencias observadas en el rendimiento académico de los estudiantes universitarios en la asignatura Introducción a la Computación, específicamente en comprender el dominio de los problemas y el desarrollo del método en la fase de Análisis Orientado a Objetos (AOO); también se consideró para su diseño la identificación, por parte de los profesores, de los pasos esenciales del AOO para la resolución de problemas. 
Respecto a las deficiencias observadas en los estudiantes, específicamente se encontró dificultad en la determinación de las operaciones aritméticas de los métodos, resolución aritmética del problema aplicando lógica computacional y la definición del tipo de estructura del método (secuencial, selectivo).

ESENCI comprende las etapas de planificación y ejecución, correspondiendo al diseño del problema computacional y las directrices para la ejecución de la resolución por parte de los estudiantes, enmarcado en el Análisis Orientado a Objetos en la fase de análisis. Esta estrategia orienta al profesor en los pasos esenciales para la enseñanza de resolución de problemas computacionales en ingeniería.

Para el diseño de la estrategia se utilizó el método de Aprendizaje Basado en Problemas (ABP), lo cual ofrece una perspectiva novedosa al ser aplicado en resolución de problemas computacionales bajo el enfoque Orientado a Objetos; este método fomenta el aprendizaje colaborativo y por descubrimiento significativo. Los criterios esenciales del método ABP integrados a ESENCI para la construcción y resolución de problemas computacionales fueron los siguientes: plantear situaciones problemáticas relacionadas con el área profesional, apropiadas al nivel cognitivo del estudiante, ámbito del problema conocido por el estudiante y la presencia de varias soluciones posibles. Respecto a los pasos del Análisis Orientado a Objeto (AOO) se incorpora a ESENCI la identificación de clases, el uso de la notación establecida para los identificadores, definir interfaces de entrada y salida, desarrollo de operaciones aritméticas, definición del tipo de estructura, y establecer el tipo y cardinalidad de la relación entre clases.

La estrategia de enseñanza propuesta contribuye con la vinculación teoría-prácticainvestigación necesaria a lo largo del desempeño profesional del estudiante del área computacional, fomentando el auto aprendizaje y el trabajo en equipo, contribuye con la planificación del diseño del problema computacional. Adicionalmente esta estrategia pretende fomentar la independencia del estudiante hacia el profesor, promover el desarrollo de competencias instrumentales, interpersonales y sistémicas, en concordancia con las Directrices para la Formulación del Perfil Profesional bajo el Enfoque de Competencias establecidos por la Universidad.

ESENCI se limita a la fase de AOO siguiendo los pasos correspondientes a la identificación de clases, atributos y métodos en el problema computacional para la resolución 
del mismo, centrándose en las etapas Planificación y Ejecución, donde el profesor define los medios y recursos a utilizar y el contenido necesario para el proceso de ejecución del problema.

Se recomienda extender ESENCI empleando las etapas de diagnóstico y evaluación en la planeación estratégica de la enseñanza de resolución de problemas computacionales en ingeniería.

\section{REFERENCIAS}

Ahumada, C., Gallegos, M. y Lozano S. (2012). Aprendizaje significativo para la asignatura de lógica computacional. XV Congreso internacional sobre innovaciones en docencia e investigación en ciencias económico administrativas, México.

Albanese, M., y Mitchell, S. (1993). Problem-based learning: A review of the literature, on its outcomes and implementation issues. Academic Medicine. 68(1), 52-81.

Álvarez, Y. y Ruiz, M. (2010). Actitudes hacia las matemáticas en estudiantes de ingeniería en universidades autónomas venezolanas. Revista de Pedagogía. 31(89), 225-249.

Arpí, C., Àvila, P., Baraldés, M., Benito, H., Gutiérrez del Moral, M., Orts, M., Rigall, R. y Rostan, C. (2012). El ABP: origen, modelos y técnicas afines. Aula de innovación educativa. 216, 14-18. Disponible: http://redined.mecd.gob.es/xmlui/handle/11162/87668[Consulta: 2014, Julio 19].

Barco, M. (2012). Formulación de un modelo para la enseñanza del ajuste por inflación fiscal como parte de un proyecto de aprendizaje de los tributos basado en el metódo ABP (Aprendizaje Basado en Problemas) para los estudiantes de la materia Sistemas Tributarios de la Universidad Centroccidental "Lisandro Alvarado”, Tesis de Pregrado. Universidad Centroccicental "Lisandro Alvarado". Barquisimeto.

Booch, G. (2001). Análisis y Diseño Orientado a Objetos con aplicaciones. México: Editorial Alhambra Mexicana.

ACM. (2013). Computing Curricula CS2013 Disponible: http://ai.stanford.edu/users/sahami/CS2013//ironman-draft/cs2013-ironman-v1.0.pdf [Consulta: 2014, Junio 19] 
Decanato de Ciencias y Tecnología (DCYT). (2012). Ingeniería en Informática. Universidad Centroccidental "Lisandro Alvarado". [Página Web en línea]. Disponible: http://dcyt.ucla.edu.ve /index.php/ carreras/tm-informatica [Consulta: 2014, Mayo5]

Díaz, F. y Hernández, G. (2002). Estrategias docentes para un aprendizaje significativo. Una interpretaciónconstructivista. México: McGraw Hill Interamericana.

Duch, B. (1996). Problems: A key factor in PBL. About Teaching: University of Delaware. Newsletter of the Center for teaching Effectiveness, About Teaching. USA.

Echavarria, M. (2010). Aplicación del Aprendizaje Basado en Problemas en Ingeniería. Revista EIA. 14, 85-95.

Esteves, M., Morgado, L., Martins, P. y Fonseca, B. (2006). The use of Collaborative Virtual Environments to provide student's contextualisation in programming.ICTE 2006.IV International Conference on Multimedia and Information and Communication Technologies in Education, España.

Feo, R. (2010). Orientaciones básicas para el diseño de estrategias didácticas. Revista Tendencias Pedagógicas. 16, 207-220. Disponible: http://www.tendenciaspedagogicas.com/Articulos/2010_16_13.pdf [Consulta: 2014, Mayo 5]

García, J. (2007). La Programación en los planes de estudio. II Simposio Nacional de Docencia en la Informática (SiNDI), Septiembre 2007. Zaragoza, España. 113-151, Disponible:

http://bioinfo.uib.es/ joemiro/aenui/ProcSindi/P2007/PonProgr.pdf[Consulta: $\quad 2014$, Julio 5]

Guevara, G. (2010). Aprendizaje Basado en Problemas como técnica didáctica para la enseñanza del tema de la recursividad. InterSedes: Revista de las Sedes Regionales. 11 (20), 142-167.

Hernández, R., Fernández, C. y Baptista, P. (2010). Metodología de la Investigación. 5ta Ed. McGraw Hill: México.

Hurtado, J. (2010). Metodología de la investigación. Guía para la comprensión holística de la ciencia. Caracas: Ediciones Quirón. CIEA Sypal.

Jofré, C. y Contreras, F. (2013). Implementación de la Metodología ABP (Aprendizaje Basado en Problemas) en Estudiantes de Primer Año de la Carrera de Educación Diferencial.Estudios Pedagógicos. 39 (1), 99-113. 
Lovos, E., González, A. y Fernández, I. (2013). Combinando ABP y herramientas colaborativas para la enseñanza de Programación en el primer año de la Licenciatura en Sistemas de la UNRN. I Jornadas Nacionales y III Jornadas de la UNC: Experiencias e Investigación en Educación a Distancia y Tecnología Educativa. Córdoba. Disponible: http://sedici.unlp.edu.ar/handle/10915/39206 [Consulta: 2014, Octubre 5]

Marieta, C., Andrés, M., León, I. (2015). Aprendizaje basado en problemas: una aplicación práctica en la enseñanza de materiales. XII Jornadas Internacionales de Innovación Universitaria. Educar para transformar: Aprendizaje experiencial. Villaviciosa de Odón, 20 y 21 de julio, 2015. Pp. 759-765.

Martínez, F. y Torres, L. (2010). El aprendizaje basado en problemas. Experiencias piloto en la enseñanza de un lenguaje de programación. Revista de Ciencias Exactas e Ingeniería.32, 1-8.

Michel, R. (2011). MCC Lumin como apoyo para disminuir los índices de reprobación. VI Foro de Investigación Educativa, México.

Morales, P. y Landa, V. (2004). Aprendizaje basado en problemas. Theoria.13, 145-157. Disponible: http://www.redalyc.org/articulo.oa?id=29901314[Consulta: 2015, Junio $10]$.

NCSS (2012). Statistical software. Disponible: www.ncss.com [Consulta: 2014, Junio 10].

Restrepo, B. (2009). Aprendizaje basado en problemas (ABP): una innovación didáctica para la enseñanza universitaria. Educación y Educadores. 8, 9-19.

Rojas, P. (2010). El Aprendizaje Basado en Problemas (ABP) como estrategia metodológica de enseñanza y aprendizaje de la integral indefinida en paralelo con derivadas y su incidencia en el rendimiento académico de los estudiantes de Ingeniería en Informática de Inacap, Chillán. Chile. Tesis de Maestría, Inacap, Chillán.

Romero, A. y García, J. (2008). La elaboración de problemas ABP. El aprendizaje basado en problemas en la enseñanza universitaria. Servicio de Publicaciones de la Universidad de Murcia. 37-55.

Sáez, P. y Monsalve, C. (2008). Aprendizaje Basado en Resolución de Problemas en Ingeniería Informática. Formación Universitaria. 1(2), 3-8.

Salgado, A., Alonso I., Gorina, A. y Tardo, Y. (2013). Lógica algorítmica para la resolución de problemas de programación computacional: una propuesta didáctica.Revista Didasc@lia: Didáctica y Educación. 4(1), 67. 
Oficina de Planificación del Sector Universitario (OPSU). (2004). Sistema de Evaluación y Acreditación. Normas para la Tramitación y Evaluación de Proyectos de Creación de Instituciones y Carreras de Pregrado. Caracas, Venezuela.

Verde, Y., Cañas, T., López, A. y Trujillo, T. (2011). Propuesta de estrategia didáctica fundamentada en el Aprendizaje Basado en Problemas para la apreciación de las Artes Plásticas. Revista Didasc@lia: Didáctica y Educación. 4, 101-116.

Villalobos, R. (2006). Mejoría del rendimiento estudiantil en Medicina Tropical con el método de Aprendizaje Basado en Problemas, en la Facultad de Medicina de la Universidad del Zulia.Kasmera. 34(2), 123-126.

Universidad Centroccidental Lisandro Alvarado (2005). Manual de Directrices para la formulación del perfil profesional bajo enfoque de competencias.Comisión de Currículo. Disponible: http://www.ucla.edu.ve/comision/curriculo/competencia.pdf [Consulta: 2014, Junio 5]

Zapata, M. y Flores, L. (2008). Identificación de los estilos de aprendizaje en estudiantes universitarios. Revista Estilos de Aprendizaje. 2 (2), 130-152. 